\title{
Impact of mobility in mobile communication systems
}

\author{
M. Zonoozi and P. Dassanayake
}

Department of Electrical \& Electronic Engineering, Victoria University of Technology,

PO Box 14428 MCMC, Melbourne, VIC 8001, AUSTRALIA.

Email:mahmood@cabsav.vut.edu.au

Fax: 6136884908 - Tel: 6136884767

\begin{abstract}
A new method is developed for systematic tracking of the random movement of a mobile station in a cellular environment. It incorporates mobility parameters under most generalized conditions, so that the model could be tailored to be applicable in most cellular environments. This mobility model is used to characterise the cell residence time of both new and handover calls occurring in a cellular mobile communication system. It is shown that the cell residence time can be described by the generalized gamma distribution.
\end{abstract}

Keywords

Handover, mobility, cell residence time

\section{INTRODUCTION}

In a cellular mobile communication network, depending on whether a call is originated in a cell or handed over from a neighbouring cell, two different cell residence times can be specified and they are the new call cell residence time and the handover call cell residence time, respectively. New call cell residence time is defined as the length of time a mobile terminal resides in the cell where the call was originated before crossing the cell boundary. Similarly, the handover call cell residence time is defined as the time spent by a mobile in a given cell to which the call was handed over from a neighbouring cell before crossing to another cell, (Figure 1). New call cell residence time, $T_{n}$, and the handover call cell residence time, $T_{h}$, are two random variables whose distributions have to be found. A literature survey shows that a relatively few in-depth papers have been published on this subject. Moreover, most of these are restricted to simple mobility cases, and often based on assumptions made 


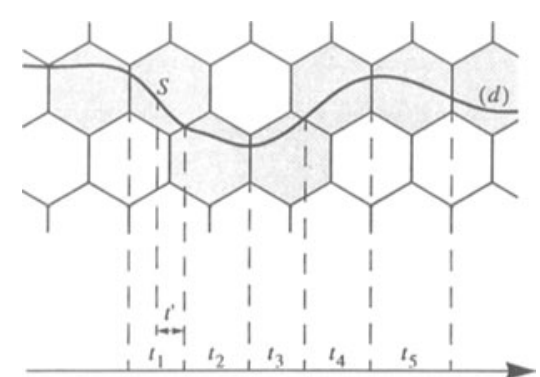

Figure 1. Cell residence times for a mobile travelling on a path of (d), $S$ is the start time of a call. $T_{h} \in\left\{t_{1}, t_{2}, t_{3}, t_{4}, t_{5}\right\} \quad T_{n} \in\left\{t^{\prime}\right\}$

without justification.

Hong and Rappaport [1] have obtained the probability density function (pdf) of a simplified case of mobility where there is no change in speed or direction of the mobile. Further, the initial speed of the mobile was assumed to follow a uniform distribution. Del Re, Fantacci and Giambene [2] have assumed that mobiles, before crossing a cell travel a distance uniformly distributed between 0 and $2 R$, where $R$ is the hexagonal cell side. They also assume a constant speed with uniform distribution and conclude that the pdf of cell residence time is different to that shown in [1]. Inoue, Morikawa and Mizumachi [3] have applied the procedure of [1] for a case of non-uniform speed distribution. However they end up with a set of unsolved integral equations. Yeung and Nanda [4], Xie and Kuek [5], Xie and Goodman [6] have shown that contrary to the assumption made in [1], the speed and direction distributions of the in-cell mobiles are different from those of the cell-crossing mobiles. A more precise distribution for the speed and direction can be obtained using their Biased Sampling formula.

While Sanchez Vargas [7], and Lue [8] have assumed cell residence time to be uniformly distributed over the call duration, Nanda [9], Lin, Mohan and Noerpel [10] have taken a general distribution for the cell residence time. For the sake of simplicity, in the absence of any proved probability distribution, many authors dealing with the mobility problem have assumed cell residence time to be an exponentially distributed random variable either explicitly or implicitly [11]-[17].

\section{MOBILITY MODELLING OF THE SIMPLIFIED CASE}

For a simplified case of mobility where there is no change in speed and direction of the mobile, cell residence time distribution can be obtained analytically. In this simplified case, the direction of the mobile at the starting point, $\alpha_{0}$, is taken to be uniformly distributed in the range $(0,2 \pi)$, and it is assumed to remain constant along its path. Moreover, the initial speed of the mobile is also taken to be a uniformly distributed random variable in the range $\left(0, V_{m}\right)$, and it is assumed to remain constant along the mobile path. Users are assumed to be independent and uniformly distributed over the entire region. Initial location of a mobile is represented by its distance $\rho_{0}$ and direction $\theta_{0}$ from the base station which is located at the centre of the cell. Let $f_{T_{n}}(t)$ denote the pdf of the new call cell residence time. Then, according to [1],

$$
f_{T_{n}}(t)=\left\{\begin{array}{lr}
\frac{8 R}{3 \pi V_{m} t^{2}}\left\{1-\left[1-\left(\frac{V_{m} t}{2 R}\right)^{2}\right]^{3 / 2}\right\} & 0 \leq t \leq \frac{2 R}{V_{m}} \\
\frac{8 R}{3 \pi V_{m} t^{2}} & t \geq \frac{2 R}{V_{m}}
\end{array}\right.
$$


A handover call starts from the boundary of a cell with mobile having a direction $\alpha_{0}$ uniformly distributed over $(-\pi / 2, \pi / 2)$. The pdf of the handover call cell residence time, $f_{T_{h}}(t)$ can be calculated in a similar manner to (1),

$$
f_{T_{h}}(t)=\left\{\begin{array}{lr}
\frac{4 R}{\pi V_{m} t^{2}}\left\{1-\left[1-\left(\frac{V_{m} t}{2 R}\right)^{2}\right]^{1 / 2}\right\} & 0 \leq t \leq \frac{2 R}{V_{m}} \\
\frac{4 R}{\pi V_{m} t^{2}} & t \geq \frac{2 R}{V_{m}}
\end{array}\right.
$$

In [5]-[6], it is shown that the speed and direction distributions of the in-cell terminals are different from those of the cell-boundary crossing terminals. Let $f_{V_{0}}\left(v_{0}\right)$ denote the pdf of the speeds of all terminals and $f_{V_{0}}^{*}\left(v_{0}\right)$ denote the pdf of the speeds of cell-boundary crossing terminals. Based on the Biased Sampling [18], the speed pdf of the cell-boundary crossing terminals can be derived [5] as,

$$
f_{V_{0}}^{*}\left(v_{0}\right)=\frac{v_{0} f_{V_{0}}\left(v_{0}\right)}{E\left[V_{0}\right]}= \begin{cases}\frac{v_{0}}{V_{m} E\left[V_{0}\right]} & 0 \leq v_{0} \leq V_{m} \\ 0 & \text { otherwise }\end{cases}
$$

Similarly, let $f\left(\alpha_{0}\right)$ be the pdf of the directions of all terminals, which has uniform density in the range $(0,2 \pi)$. Based on the Biased Sampling, the pdf of the directions of the cell-boundary crossing terminals, $f^{*}\left(\alpha_{0}\right)$, can be obtained as,

$$
f^{*}\left(\alpha_{0}\right)= \begin{cases}\frac{1}{2} \cos \left(\alpha_{0}\right) & -\frac{\pi}{2} \leq \alpha_{0} \leq \frac{\pi}{2} \\ 0 & \text { otherwise }\end{cases}
$$

Equation (4) shows that the pdf of the direction of the cell-crossing terminals is not uniform, but has a direction bias towards the normal. Considering (3) and (4), the relations for $f_{T_{n}}(t)$ and $f_{T_{h}}(t)$ could be modified accordingly.

\section{MOBILITY MODELLING FOR GENERAL CASE}

Eqs. (1)-(2) represent new and handover call cell residence time distributions for the simplified case of mobility where there is no change in speed or direction and no biasing in speed or direction of boundary crossing mobiles. In the general case, the mobility modelling should include changes in direction and speed of the mobile. Moreover, it is unrealistic to assume that the speed is uniformly distributed and remains constant. Extension of the analysis of the simplified case to cover the general case is virtually impossible, and simulation appears to be the only way out. The simulation model is aimed at obtaining statistical estimates of the mobile cell boundary crossings in a cellular environment where the mobile is allowed to move freely with randomly varying velocities and directions within realistic bounds. A uniform distribution is assumed for spatial location of the users. This assumption is valid, since throughout a cellular network, the relative orientation of streets and cells varies somewhat randomly, giving on the average a nearly uniform distribution of possible directions [6]. Since the destination point of the mobiles can be any place in the coverage area, mobiles are allowed to move away from the starting point in any direction with equal probability. Therefore, a uniform distribution in the range is suitable for the initial mobile direction. Depending on the structure of the cellular mobile coverage area, a mobile may move towards the destination point via 

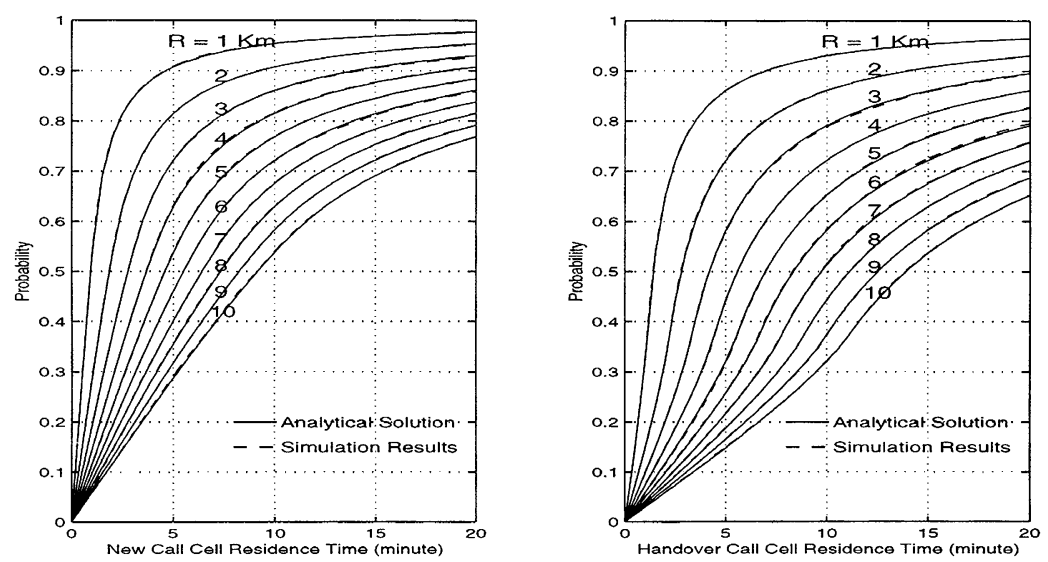

Figure 2. Comparison between simulation and analytic solution for the special case

different paths. However, in any case, the mobile direction is biased towards destination to prevent it from circling around. In the simulation model, the initial mobile direction is taken to be uniformly distributed in the cell area, and the directions at successive steps are allowed to change within a set bound referred herein as drift.

The probability distribution of the variation of the mobile direction along its path is taken to be uniform and the variation is taken to be in the range $(-\alpha,+\alpha)$ degrees with respect to the current direction. The value of $\alpha$ is chosen, depending on the street structure of the cell area, to be a low value for the cells with more straight streets and a high value for the cells with less straight streets. The effect of $\alpha$ on the probability of boundary crossing can be verified by comparing different values of $\alpha$ with respect to a reference. The initial speed of a mobile unit, at the instance the call is initiated, is taken as a random variable with truncated Gaussian pdf, $f_{V_{0}}\left(v_{0}\right)$, having a mean and standard deviation of $\mu_{v}$ and $\sigma_{v}$, respectively. The choice of such a distribution seems reasonable, since the more extreme the speed value, the less likelihood of its occurrence. Also, it is unlikely that the speed exceeds a certain maximum value.

At any time instant, the mobile speed is a random variable correlated with the previous speed, $v_{p}$. The current speed, $v_{c}$, of each mobile is taken to be a uniformly distributed random variable in the range $\pm 10 \%$ of the previous speed. Any increase in the speed above $100 \mathrm{Km} / \mathrm{h}$ is not allowed, and the minimum speed is taken to be $0 \mathrm{Km} / \mathrm{h}$.

In order to check the validity of the proposed simulation model a test run is made for the simplified case described in Section 2, with the same assumptions held. The probability distribution function of the cell residence time is calculated through (1)-(2) and compared with the results obtained by the simulation. As shown in Figure 2 the simulation results are in good agreement with the analytical results.

\section{CELL RESIDENCE TIME DISTRIBUTION}

What is of important here is not the actual mobile trajectories, but the distribution of the users' cell residence time. With this in mind, we wish to test the hypothesis that the new call and handover call residence time data follows a particular probability distribution. Following [19], we proceed with the generalized gamma distribution which provides a series of pdf of the form 
$f_{T}(t ; a, b, c)=\frac{c}{b^{a c} \Gamma(a)} t^{a c-1} e^{-\left(\frac{t}{b}\right)^{c}} \quad t, a, b, c>0$

where $\Gamma(a)$ is the gamma function, defined as $\Gamma(a)=\int_{0}^{\infty} x^{a-1} e^{-x} d x$, for any real and positive number $a$. The parameters $a, b, c$ can be classified on the basis of their physical or geometric interpretation, as one of the three types, namely location, scale and shape. The evaluation of the agreement between the distributions obtained by simulation and the best fitted generalized gamma distribution is done by using the Kolmogorov-Smirnov goodness-of-fit test. Given the generalized gamma distribution as the hypothesized distribution, the values of the parameters $a, b, c$ are found such that the maximum deviation to be a minimum. The maximum deviation shows the biggest divergence between the observed and the hypothesized distributions. The results show that the values of $a$ and $c$ are constant and are independent of cell size, while $b$ varies with the cell size according to

$b \approx \begin{cases}1.84 R & \text { new call } \\ 1.22 R & \text { handover call }\end{cases}$

\section{MEAN CELL RESIDENCE TIME}

The mean cell residence time for the new and the handover calls can be found by

$$
\begin{aligned}
E\left[T_{n}\right] & =\int_{0}^{\infty} t \cdot f_{T_{n}}(t) d t \\
E\left[T_{h}\right] & =\int_{0}^{\infty} t \cdot f_{T_{h}}(t) d t
\end{aligned}
$$

Yeung and Nanda [4], have shown that for an arbitrary speed pdf and zero drift the mean cell residence time can be obtained through the following equations

$$
\begin{aligned}
& E\left[T_{n}\right]=\frac{8 R E[1 / V]}{3 \pi} \\
& E\left[T_{h}\right]=\frac{\pi R}{2 E[V]}
\end{aligned}
$$

where $R$ is the cell radius and $V$ is the speed of the mobile in the cell. A comparison of the results obtained from (9)-(10) with (7)-(8) assuming generalized gamma pdf for $f_{T_{t}}(t)$ and $f_{T_{t}}(t)$ shows that the maximum difference (error) is less than $0.0007 \%$ in the case of new calls and $0.0021 \%$ in the case of handover calls.

\section{EFFECT OF CHANGE IN DIRECTION AND SPEED}

Depending on the street structure, a mobile can move in different paths and may possess different speeds. The extent of mobile change in direction (drift) and change in speed are the two parameters that govern its mobility. The effect of change in direction or speed of mobiles can be considered as equivalent to a change in an average distance travelled or time spent in the cell, before moving out. Any increase in mobile's drift can be treated as contributing to an effective increase in the cell radius. Similarly, any increase in speed of the mobile can be 
treated as contributing to a decrease in the cell residence time which can be interpreted as an effective decrease in the cell size. Therefore, cells with a broad variety of mobility parameters can be replaced by an equivalent reference cell with an effective radius. A reference cell is defined as a cell with the following mobility parameters, (a) mobile moves in a straight path, i.e. $\alpha=0^{\circ}$, (b) initial speed of a mobile follows a truncated Gaussian pdf with an average of $\mu_{v}=50[\mathrm{Km} / \mathrm{h}]$ and standard deviation of $\sigma_{v}=15[\mathrm{Km} / \mathrm{h}]$.

Our aim is to relate cells with given mobility parameters (i.e. drift $\alpha$ and average speed $v$ ) to the reference cell. We consider two different cases

case i.) cells having mobility parameters defined by a uniformly distributed drift pdf in the range $\left(-k^{\circ}, k^{\circ}\right)$ and speed pdf similar to the reference cell.

case ii.) cells having mobility parameters defined by zero drift (similar to the reference cell) and a truncated Gaussian speed pdf with an average value of $v \neq \mu_{v}$ and standard deviation of $\sigma_{v}=(v-5) / 3[\mathrm{Km} / \mathrm{h}]$.

Consider a cell with the radius of $R_{\alpha}$ where its mobility parameters are according to case $i$. The radius of the equivalent cell (which has the same residence time) with mobility parameters of the reference cell, $\Re_{\alpha}$, is given by $\Re_{\alpha}=R_{\alpha}+\Delta R_{\alpha}$, where $\Delta R_{\alpha}$ is the excess cell radius required to replace $R_{\alpha}$ (i.e. radius of a cell where its mobiles can move around with a uniformly distributed drift pdf in the range $\left(-k^{\circ}, k^{\circ}\right)$ ) with $\Re_{\alpha}$ (i.e. radius of an equivalent cell where its mobiles can move on a straight line). The data obtained by simulation satisfy the empirical equation of (11) in a least mean square sense.

$\Delta R_{\alpha}=0.0038 k R_{\alpha}$

Therefore the equivalent cell radius will be

$\Re_{\alpha}=K_{\alpha} R_{\alpha}$

where $K_{\alpha}$ is the proportionality factor and is equal to $(0.0038 k+1)$. In the same manner, consider a cell with the radius of $R_{v}$ where its mobility parameters are according to case ii. The radius of an equivalent reference cell, $\Re_{v}$, which has the same cell residence time is given by $\Re_{v}=R_{v}+\Delta R_{v}$, where $\Delta R_{v}$ is the excess cell radius required to replace $R_{v}$ with $\Re_{v}$. The data obtained by simulation satisfy the empirical equation of (13) in a least mean square sense.

$\Delta R_{v}=\left(\frac{\mu_{v}}{v}-1\right) R_{v}$

Therefore the equivalent cell radius will be

$\Re_{v}=K_{v} R_{v}$

where $K_{\nu}$ is the proportionality factor and equals to $\left(\mu_{v} / v\right)$. In a case where both drift and speed are different from those of the reference cell, the equivalent value of the cell radius, $\Re_{\alpha v}$, for a cell of radius, $R_{\alpha \nu}$, can be calculated by the following relation, $\left(R_{\alpha \nu}\right.$ is the cell radius for a cell which supports mobility parameters of $\alpha$ and $v$ )

$\Re_{\alpha \nu}=K_{v} K_{\alpha} R_{\alpha \nu}$

Therefore, in a cell of radius $R_{\alpha \nu}$, the gamma distribution parameter $b$ for a mobile with an average speed $v$ and a drift $\alpha$ in the range $\left(-k^{\circ}<\alpha<k^{\circ}\right)$ can be described as 
$b \approx \begin{cases}1.84 \Re_{\alpha \nu} & \text { new call } \\ 1.22 \Re_{\alpha \nu} & \text { handover call }\end{cases}$

\section{CONCLUSIONS}

This paper presented a methodology appropriate for mobility modelling of users in a cellular mobile communication system. The proposed model traces mobiles systematically in a cellular environment where they are allowed to move in a quasi-random fashion with assigned degrees of freedom. This model enables the development of a computer simulation algorithm that provides statistical estimates of the cell boundary crossing features and hence the characterisation of cell residence times. Results show that the generalized gamma distribution is a good approximation for the cell residence time distribution of both new and handover calls. It is also shown and that the negative exponential distribution is a good approximation for the channel holding time distribution in cellular mobile systems.

It was also shown that an increase in mobile drift can be treated as contributing to an effective increase in the cell radius. Similarly, it was shown that an increase in the speed of a mobile can be treated as contributing to a decrease in the cell size, and vice versa. Taking this excess cell radius into account for different values of drift and speed, a broad variety of cell areas with different street orientations and traffic flows can be handled by this mobility model.

\section{ACKNOWLEDGEMENTS}

The authors gratefully acknowledge the support provided by the Australian Telecommunications and Electronics Research Board (ATERB) for this project. The first author also gratefully appreciates the support provided by the Telecommunication Company of Iran.

\section{REFERENCES}

[1] D. Hong, S. S. Rappaport, 'Traffic model and performance analysis for cellular mobile radio telephone systems with prioritized and nonprioritized handoff procedures', IEEE Transactions on Vehicular Technology, vol. 35, no. 3, pp. 77-92, 1986.

[2] E. Del Re, R. Fantacci, G. Giambene, 'Handover and dynamic channel allocation techniques in mobile cellular networks', IEEE Transactions on Vehicular Technology, vol. 44, no. 2, pp. 229-237, May 1995.

[3] M. Inoue, H. Morikawa and M. Mizumachi, "Performance analysis of microcellular mobile communication systems," 44th. IEEE Vehicular Technology Conference, Stockholm, pp. 135-139, Jun. 1994.

[4] K. L. Yeung and S. Nanda, "Optimal mobile-determined micro-macro cell selection," 6th. IEEE International Symposium on Personal, Indoor and Mobile Radio Communications (PIMRC'95), Toronto, pp. 294-299, Sep. 1995.

[5] H. Xie, S. Kuek, "Priority handoff analysis," 43rd. IEEE Vehicular Technology Conference, New Jersey, pp. 855-858, May 1993.

[6] H. Xie, D. J. Goodman, "Mobility models and biased sampling problem," 2nd. IEEE International Conference on Universal Personal Communications, pp 804-807, 1993.

[7] J. H. Sanchez Vargas, "Teletraffic performance of cellular mobile radio systems," Ph.D dissertation, University of Essex, England, 1988.

[8] X. Lue, 'Investigation of traffic performance in mobile cellular communication systems' Master thesis, University of Melbourne, Australia, 1991. 
[9] S. Nanda, "Teletraffic models for urban and suburban microcells: cell sizes and handoff rates," IEEE Transactions on Vehicular Technology, vol. 42, no. 4, pp. 673-682, Nov. 1993.

[10] Y. B. Lin, S. Mohan, A. Noerpel, "Queuing Priority channel assignment strategies for PCS handoff and initial access," IEEE Transactions on Vehicular Technology, vol. 43, no. 3, pp. 704-712, Aug. 1994.

[11] C. Purzynski and S. S. Rappaport, "Multiple call hand-off problem with queued hand-offs and mixed platform types," IEE Proceedings on Communications, vol. 142 no 1, pp. 31-39, Feb. 1995.

[12] C. Purzynski and S. S. Rappaport, "Traffic performance analysis for cellular communication systems with mixed platform types and queued hand-offs," 43rd. IEEE Vehicular Technology Conference, New Jersey, pp. 172-175, May 1993.

[13] M. Naghshineh and A. S. Acampora, "Design and control of micro-cellular networks with QOS provisioning for real-time," 3rd. IEEE International Conference on Universal Personal Communications, San Diego, pp 376-381, Sep. 1994.

[14] S. S. Rappaport, "The multiple-call handoff problem in high capacity cellular communications systems," IEEE Transactions on Vehicular Technology, vol. 40, no. 3, pp. 546-557, Aug. 1993.

[15] W. M. Jolley, R. E. Warfield, "Modelling and analysis of layered cellular mobile networks," 13th International Teletraffic Congress, pp. 161-166, 1991.

[16] H. Jiang and S. Rappaport, "Handoff analysis for CBWL schemes in cellular communications," 3rd. IEEE International Conference on Universal Personal Communications, San Diego, pp 496-500, Sep. 1994.

[17] S. Rappaport, L. R. Hu, "Microcellular communication systems with hierarchical macrocell overlays: traffic performance models and analysis," Proceedings of IEEE, vol. 82, no. 9, pp. 1383-1397, Sep. 1994.

[18] D. R. Cox and P. A. W. Lewis, The statistical analysis of series of events. London: Chapman and Hall, 1978.

[19] A. M. Law, W. D. Kelton, Simulation modelling and analysis. New York: McGraw-Hill, 1991.

\section{BIOGRAPHIES}

Mahmood Zonoozi received the B.Sc. and M.Sc. degrees in electrical engineering from K. $\mathrm{N}$. Toosi University, Iran. He has worked in satellite and microwave communication departments of Iran Telecom for several years. He is currently working towards the $\mathrm{PhD}$ degree at the Victoria University of Technology, Melbourne, Australia. He is attached to the Mobile Communications and Signal Proccesing Research Group of the university, and his project deals with the issues involved in handover of cellular mobile communication systems.

Prem Dassanayake received his B.Sc. Eng degree from University of Sri Lanka, M.Sc. and $\mathrm{PhD}$ degrees from University of Wales, Cardiff, U.K. At present he is attached to the Department of Electronics and Electrical Engineering of the Victoria University of Technology, Footscray, Australia. Prior to joining Victoria University Dr. Dassanayake has worked at the University of Moratuwa, Sri Lanka and the University of Bahrain, Bahrain. He has also been a visiting researcher at the Bureau of Medical Devices, Health and welfare, Ottawa, Canada and at Telstra Research Laboratories, Clayton, Australia. 